Original Research Paper

\title{
Experimental Investigation on the Failure Mechanism of Bovine Bone by Three-point Bending Tests
}

\author{
${ }^{1,2} \mathrm{Hua} \mathrm{Li},{ }^{2}$ Rujie Gao, ${ }^{2}$ Ruiqi Li, ${ }^{2} \mathrm{Xia}$ An and ${ }^{2}$ Yufeng Zhao \\ ${ }^{1}$ Department of Physical Education and Research, Northeast Petroleum University, Daqing, 163318, China \\ ${ }^{2}$ School of Physical Education, Yangtze University, Jingzhou, 434023, China
}

\author{
Article history \\ Received: 27-04-2020 \\ Revised: 02-06-2020 \\ Accepted: 09-07-2020 \\ Corresponding Authors: \\ Hua Li \\ Department of Physical \\ Education and Research, \\ Northeast Petroleum \\ University, Daqing, 163318, \\ China \\ and \\ School of Physical Education, \\ Yangtze University, Jingzhou, \\ 434023, China \\ E-mail: lihua_01@163.com
}

\begin{abstract}
Bone damage is a common type of sport injury. The loading rate affects the type of fracture and the degree of soft tissue injury. In view of the problem of whole bone bending damage and failure, this paper introduces the test method of bovine bone bending and the mechanism of bending failure under different loading rates is analyzed through experimental research and theoretical analysis. The results of three-point bending failure test of bovine bone are given and the influence of loading rate on bending failure is discussed. The results show that there are three main deformation and failure modes of bovine bone under different loading rates, namely the mode of fracture failure under high loading rate, the mode of shear failure under medium loading rate and the mode of local wedge failure under low loading rate. With the increase of loading rate, the greater the failure force is applied on the bone, the smaller the destructive deflection of the bone will be. The maximum strain of bone decreases with the increase of loading rate, while the flexural modulus of bone increases gradually, which indicates that the loading rate has an effect on the brittleness of the whole bone. For the bending model of the whole bovine bone, the prediction result of elliptical cross-section method is more in accordance with the actual situation than that of circular cross-section method.
\end{abstract}

Keywords: Biomechanics, Bovine Bone, Bending Test, Mechanical Properties, Brittleness

\section{Introduction}

The daily activities and movements of the human body are actually the process of the bones subjected to various loads. Bone has the functions of supporting the body, protecting organs and completing movement. Like many other solid materials, bone will be damaged when it is subjected to heavy loads and rapid loading and the bones are active and can be repaired by bone tissue regeneration in a certain range, which is different from general artificial materials. If the damage of bone is too large, too fast or accumulated beyond the repair ability, the bone will break. Bones have different failure strength or damage threshold under different stress levels. Its damage is a common type of sports injury for athletes due to severe exercise and collision (Bai et al., 1999; Fan and Wang, 2006). As a kind of mechanical structure, bone is in the field of mechanics and its damage is mainly due to the action of force. As a solid structure, bone is a research hotspot in the field of biomechanics (Cui and
Liu, 1997; 1998). Therefore, it is of great significance to study the mechanical behaviour of bone for the prevention and treatment of bone diseases and sports injury.

Bone is a multi-phase, lamellar and discontinuous composite structure (Sun et al., 2018). There are many aspects to study the mechanical behaviour of bones (Szabó and Thurner, 2013; Chen et al., 2015), including: (1) Stress types, such as compression, tension, bending and torsion. (2) loading methods, such as cyclic, static and dynamic loads. Due to the special structural characteristics of bone materials, their mechanical properties are more complicated and many scholars regard the bone as a type of solid material. Like other solid materials, bone will be damaged and fractured under bending loads. Flexural strength is one of the main testing index of bone mechanical properties to evaluate bone strength (Ayagara et al., 2019). Compared with the tensile strength, the flexural strength is simple and easy to obtain and the test success rate is high in the laboratory (Ferreño et al., 2017; Mumtaz et al., 2020). Bone bending test is divided into whole bone 
bending test and standard specimen bending test with the rectangular cross section. Due to the heterogeneity of bone material, the bending strengths of the standard samples are different even if they are taken from the same bone in different parts. In addition, the directionality of the standard sample has a great influence on the bending strength. The bending strength of the sample parallel to the bone axis is significantly greater than that of the specimen perpendicular to the bone axis. The bending strength measured by the whole bone experiment and the standard sample experiment is also different. The mechanical properties of the whole bone refer to the mechanical properties of the whole structure, which are not only related to the material properties of the bone, but also affected by the geometric characteristics of the bone, such as span, shape and more. The bending test is an important method for evaluating the mechanical properties of whole bone. For the traditional animal bones, the bones of cattle, especially the long bones, are often used as experimental objects to study the mechanical properties of bones due to low ethical requirements and easy handling and sampling (Liebschner, 2004; Yang, 2016). Although some studies investigating the mechanical properties of whole bones, the mechanical properties of cattle bones in different regions, different breeds and different ages are quite different, especially due to changes in nutritional conditions and food structure, the mechanical properties of bones will also change. Bovine bone is widely used in biomechanical tests and its samples used in biomechanical tests include three-point bending test, four-point bending test, compression test and torsion test. Bone fracture is often caused by impact, involving high loading rates. Many studies that have investigated the effect of loading rate on bone mechanical properties yields conflicting results and these include (Sun et al., 2018; Khor et al., 2018; Pithioux et al., 2004; Ferreira et al., 2006; Hansen et al., 2008): (a) An increase in elastic modulus, failure strain and stress with an increase in loading rate, (b) a decrease and no trend in elastic modulus and (c) a decrease in the trend for the failure parameters. The loading rate of bone is one of the necessary prerequisites for bending test. The loading rate of bone bending test reported is also different (Raftopoulos et al., 1993; Szabó et al., 2011; Chen et al., 2006). As mentioned, standard specimen of bone are mostly tested through bending tests under different loading rate for different purposes (Yang, 2016). Nevertheless, for the moment little attention has been paid to investigate the failure mechanism and failure parameter change of long whole bone by the influence of loading rate. Whether loading rate has an effect on the whole bending fracture of bovine bone and the degree of influence is still unclear.

The femur, as one of long bones, can be selected for bending test, which has important value in sports biomechanics and biomedical engineering. Three-point bending test of the whole bone is one of biomechanical test methods for bone injury (Ridha and Thurner, 2013). The aim of this paper is to determine the influence of the loading rate on failure characteristics, model and parameters of the cattle bones with the same breed, age, nutritional condition and food structure (Mumtaz et al., 2020). Taking the bovine femur as a special research topic, the effect of loading rate on the characteristics of bovine bone and fracture is discussed by analysing the results of bending test. Through threepoint bending test, the bending failure mechanism of the whole bone is discussed.

\section{Materials and Methods}

\section{Test Sample}

In order to study the biomechanical response of human bones, it is better to use human bone samples, but human bone samples are not easy to obtain, especially fresh bone samples. Compared with human bone, the ethical requirements of animals are also low and animal bones are easier to control, especially the variable of age. Bovine bones, especially bovines shown in Fig. 1, are often used as experimental samples to study the mechanical properties of bones, which is generally viewed as an acceptable surrogate for human bone (Chen et al., 2015). Bovine bones are selected mainly because: (a) The researcher has relatively little further ethical requirement to meet; (b) the close relationship between the mechanical properties of bovine bone and human bone; (c) large size makes it easy to test the whole bone in large-size.

Bovine bones are obtained directly from an animal farm with the same cattle's type, sex, age and nutritional conditions and the pairing bones from healthy adult cattle with little size differences are selected. Six pairs of fresh bovine femurs are taken within $6 \mathrm{~h}$ and the soft tissues such as muscle wrapped in bone are removed and then stored 1 day at $-20^{\circ} \mathrm{C}$ after marking. Before experiment, the bovine bone is thawed in $0.9 \%$ saline solution at room temperature for $2 \mathrm{~h}$, which are shown in Fig. 2. The two ends and the middle part of each sample are measured accurately by micrometer. The middle part of the bovine femur can be regarded as a hollow tube (Fig. 1). The statistical results of the geometric size of the bone samples Table 1 .

\section{Test Method}

There are many kinds of tests to measure the bending strength of bones, in which three-point bending test and four-point bending test are commonly used. Figure 3 shows the schematic diagram of four point bending test, which requires the same force $F$ at each loading point. It is easy to process standard bone sample into rectangular cross-section beam. Because the whole bone is not the ideal standard specimen, it is difficult to achieve the 
completely equal force $F$ at two loading points for the whole bon (Draper and Goodship, 2003). Three-point bending test is widely used in engineering field because of its simple operation and little interference from other factors. In this study, three-point bending test method is recommended for the investigation of bending failure of the whole bovine bone. In the bending test, the lower side of the bone causes tension stress and the upper side causes compression stress. The whole bovine bone is considered as a beam. Bone fracture often occurs on the side of tension stress, the stress in the outer layer of the bone is the largest, while the central axis is not affected by stress.

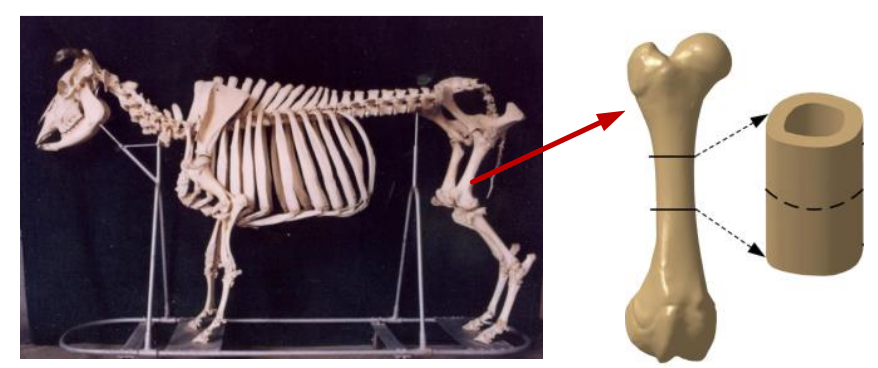

Fig. 1 Schematic diagram of the bovine bone

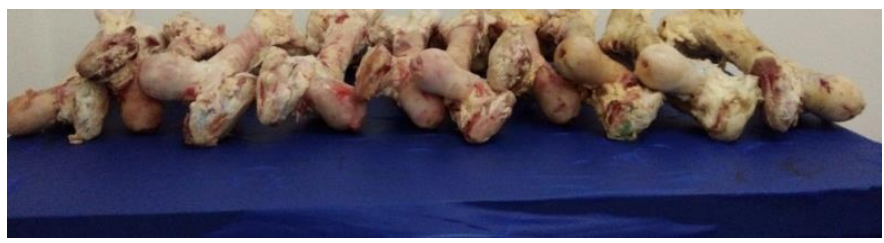

Fig. 2: Test samples of bovine femur

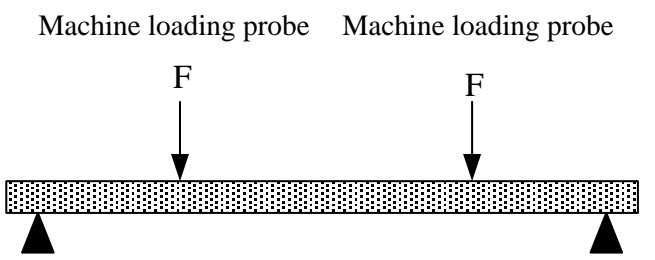

Support

(a)

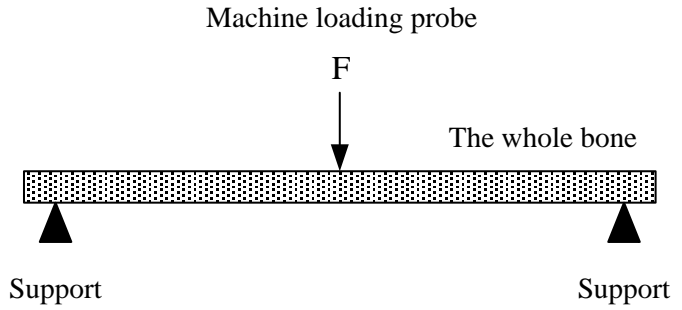

(b)

Fig. 3: Schematic diagram of bovine bone bending beam test; (a) Four point method; (b) Three point method

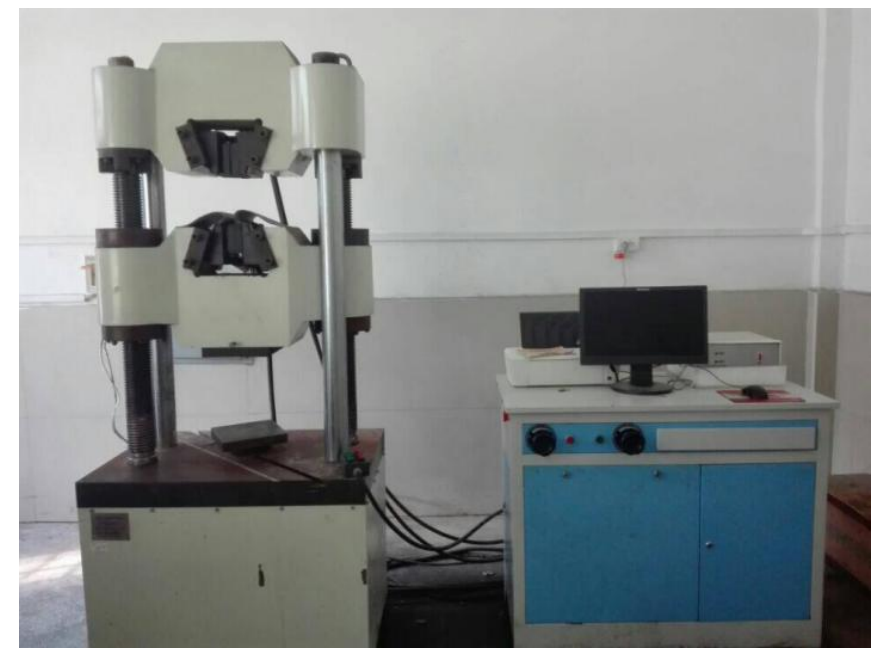

Fig. 4: Microcomputer servo control hydraulic universal testing machine 


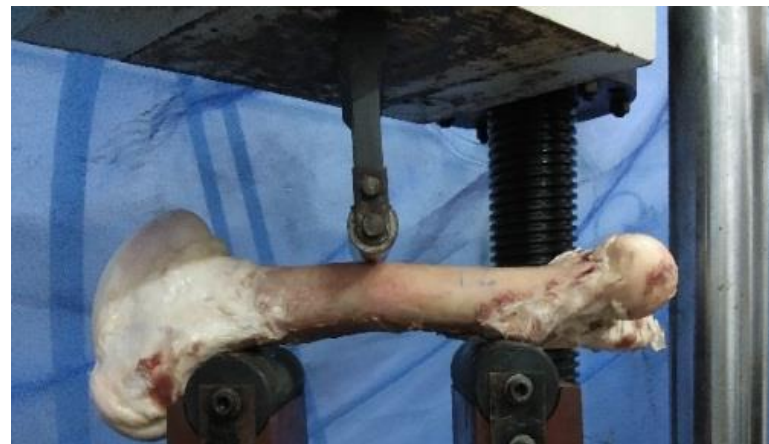

(a)

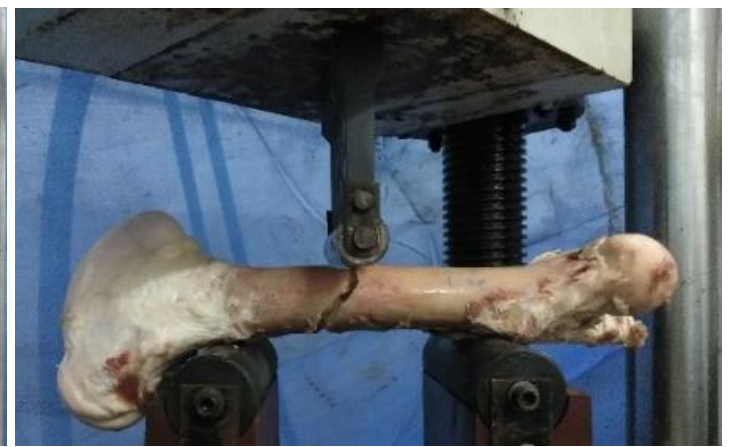

(b)

Fig. 5: Failure characteristics of bending testing for sample 1-2; (a) Before failure; (b) After failure

Table 1: Statistical results of geometric parameters of femoral samples

\begin{tabular}{|c|c|c|c|c|c|c|c|}
\hline Number & $\begin{array}{l}\text { Total } \\
\text { length } / \mathrm{mm}\end{array}$ & $\mathrm{Span} / \mathrm{mm}$ & $\begin{array}{l}\text { Outer long } \\
\text { axis/mm }\end{array}$ & $\begin{array}{l}\text { Outer short } \\
\text { axis/mm }\end{array}$ & $\begin{array}{l}\text { Maximum value } \\
\text { of thickness } / \mathrm{mm}\end{array}$ & $\begin{array}{l}\text { Minimum value } \\
\text { of thickness/mm }\end{array}$ & $\begin{array}{l}\text { Loading rate/ } \\
(\mathrm{mm} / \mathrm{min})\end{array}$ \\
\hline $1-1$ & 397.5 & 180.0 & 47.5 & 43.5 & 11.0 & 8.5 & 0.1 \\
\hline $2-1$ & 406.2 & 180.0 & 46.2 & 41.9 & 10.1 & 7.6 & 0.5 \\
\hline $\begin{array}{l}3-1 \\
3-2\end{array}$ & 380.0 & 180.0 & 45.7 & 41.2 & 10.4 & 8.1 & 1.0 \\
\hline $\begin{array}{l}4-1 \\
4-2\end{array}$ & 383.7 & 180.0 & 45.9 & 41.3 & 10.7 & 7.6 & 5.0 \\
\hline $\begin{array}{l}5-1 \\
5-2\end{array}$ & 423.6 & 180.0 & 48.1 & 43.3 & 11.9 & 8.3 & 10 \\
\hline $6-1$ & 416.1 & 180.0 & 47.9 & 42.6 & 11.4 & 7.9 & 90 \\
\hline
\end{tabular}

\section{Test Equipment and Procedures}

Because the shape, size and material properties of bone vary greatly in different parts and animal species, there is no unified test standard considering various factors in the bone biomechanical test. Figure 4, the bovine bone bending test device is a microcomputer servo controlled hydraulic test machine system.

The quasi-static bending test is mainly to study the response behavior of the structure and deformation and failure law of the whole bone under transverse loads. According to Fig. 3b, the whole bovine bone is placed on two horizontal supports with a span of $180 \mathrm{~mm}$ and force $F$ is applied at the central single point. Due to the continuous increasing of force $F$, the whole bone gradually deforms until the bending failure. The bone state before and after bending failure is shown in Fig. 5. A series of bovine bone bending tests are carried out with the displacement control method, which is shown in Table 1. Different loading rates are applied to the whole bone with a specific speed.

\section{Three Point Bending Failure Theory of the Whole Bone}

The whole bone is considered as a hollow solid beam model Fig. 6. According to the theory of material mechanics, the restraint reaction forces at the two supports of the three point bending beam are defined as follows (Draper and Goodship, 2003):

$P=\frac{F}{2}$

where, $F$ is the corresponding concentrated force at the machine loading probe.

The bending moment at the middle position of the bone is as follows (Draper and Goodship, 2003):

$M=\frac{P L}{2}$

where, $L$ is the span of the beam.

The whole bone is considered as a beam, the flexural stresses of the cross-section in middle position can be defined as (Gao et al., 2018):

$\sigma=\frac{M c}{I}$

where, $c$ is the distance from the outer edge of the bone to the neutral axis and $I$ is the moment of inertia of the beam section. 

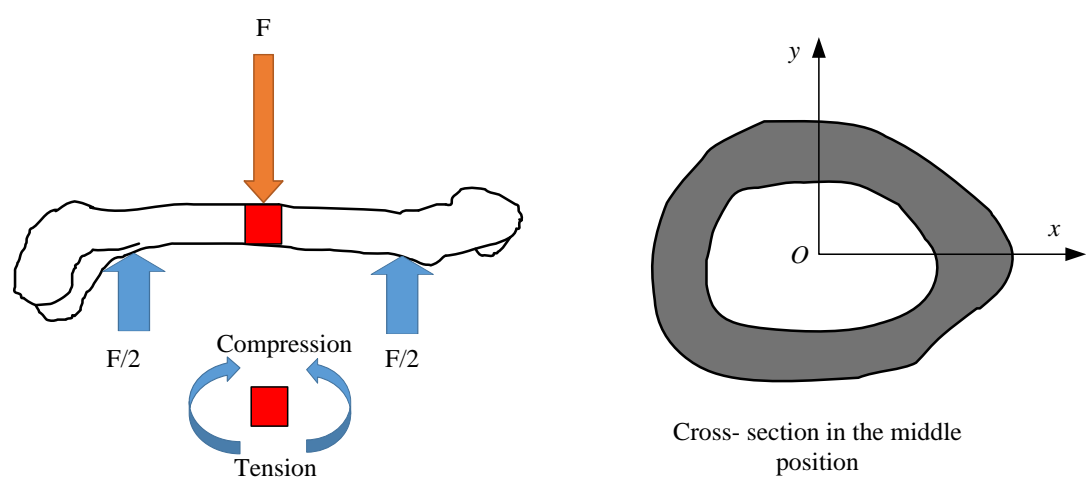

Fig. 6: Loading mode and fracture shape of bovine bone in three-point bending test

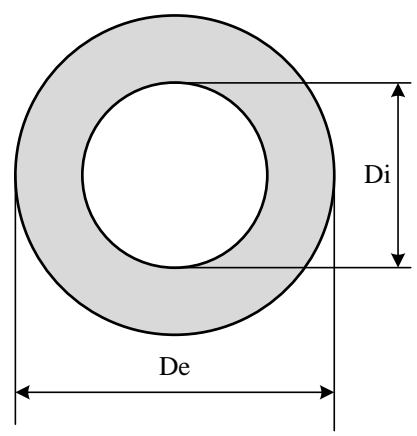

(a)

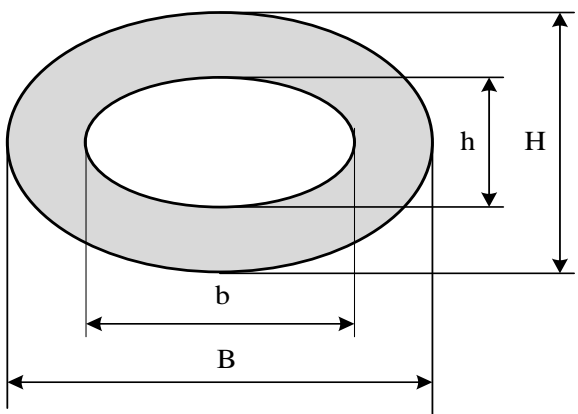

(b)

Fig. 7: Schematic diagram of calculating parameters of bone cross section; (a) Circular section; (b)Elliptic section

The key to calculate the ultimate bending stress of the whole bone is the moment of inertia of the bone section. Due to the shape section irregularity of the bone, the cross-section of the whole bone can be assumed as circular or hollow ellipse (van Lenthe et al., 2008; Kourtis et al., 2014) Fig. 7.

The formula for calculating the moment of inertia corresponding to the circular section of bone is given as follows (Mumtaz et al., 2020):

$I=\frac{\pi\left(D_{e}^{2}-D_{i}^{2}\right)}{64}$

where, $D_{e}$ is the outer diameter of the bone and $D_{i}$ is the inner diameter of the bone.

When the cross-section of the whole bone is regarded as an elliptical tubular shape, the section parameters can be obtained as follows: (a) The outer long and short axis of the middle section can be accurately measured by an electronic vernier caliper before test; (b) the inner long and short axis can be measured after the failure of bones. The moment of inertia of hollow elliptical section is (Draper and Goodship, 2003):

$$
I=\frac{\pi\left(B H^{3}-b h^{3}\right)}{64}
$$

\section{Where:}

$B=$ The outer long axis of the bone cross section

$H=$ The outer short axis

$b=$ The inner long axis

$h=$ The inner short axis of the bone cross section

To calculate the ultimate bending stress, strain and flexural modulus according to the force-displacement curves, the maximum force in the force-displacement curve is defined as the failure point. Then the ultimate bending stress, bending strain and flexural modulus of the bone are respectively as follows (Cui and Liu, 1997; 1998; Jungmann et al., 2011; Mumtaz et al., 2020):

$$
\begin{gathered}
\sigma_{f}=\frac{F_{\max } L H}{8 I} \\
\varepsilon_{f}=\frac{12 d H}{2 L^{2}} \\
E=\frac{F_{\max } L^{3}}{48 I d}
\end{gathered}
$$

where, $F_{\max }$ is the maximum force in the forcedisplacement curve and $d$ is the mid-span deflection corresponding to the maximum force. 


\section{Analysis of Test Results}

A series of three point bending beam tests are carried out under the same span with different loading rates. The loading rates are set as $0.1,0.5,1,5,10$ and $90 \mathrm{~mm} / \mathrm{min}$ respectively.

\section{Bending Failure Mechanism of the Whole Bovine Bone}

The failure characteristics of bovine bone under different loading rates are shown in Fig. 8. The bending load is applied to the sample through a small roller perpendicular to the axis of the whole bovine bone. The loading rate affects the bending failure process of bones and the failure modes can be divided into three types: (a) Tensile failure, (b) shear failure and (c) wedge-shaped local failure.

When the loading rate is higher than $10 \mathrm{~mm} / \mathrm{min}$, the bending failure mode of the whole bone shows the typical tensile damage characteristic of brittle fracture. The fracture observation shows that the macroscopic crack is an approximate flat fracture caused by the bending stress on the lower side. The fracture cross section occurs at the loading position and is substantially perpendicular to the axial direction of the femur. When the loading rate is moderate $(1-5 \mathrm{~mm} / \mathrm{min})$, the bending failure mode of the whole bone shows the characteristics of shear failure. The bending stress and shear stress develop simultaneously in the bending bone. The coupling effect of bending and shearing produces inclined shear fracture. The failure position of the upper edge of the femur occurs in the loading position and the failure position of the lower edge deviates from the vertical loading direction, which results in the inclined intersection of the fracture section and the femoral axis. When the loading rate is less than $1 \mathrm{~mm} / \mathrm{min}$, the loading roller gradually contacts with the bone and gradually presses into the bone with time, forming a wedge shape, that is, a local pressure pit. Thereafter, with the increase of force, local crushing occurs, cracks gradually expand and ultimately longitudinal macro-cracks occur, leading to bone destruction.

For the tensile failure mode, the bending deformation of the whole bone can be divided into two stages, namely the bending stage and the collapse stage (Ridha and Thurner, 2013). Due to the high loading rate, the force increases fast, the local press-in process is not obvious and the bending deformation plays a leading role. The tensile stress is generated at the lower edge of the femur after instant contact between the loading roller and bone and then the stress rate in the beam increases rapidly (Fig. 9). The mineral crystals and collagen fibers in bone are not allowed to slide sufficiently (Chen et al., 2015) and then brittle failure occurs before non-linear deformation. The faster the loading rate is, the shorter the duration of collapse stage is.

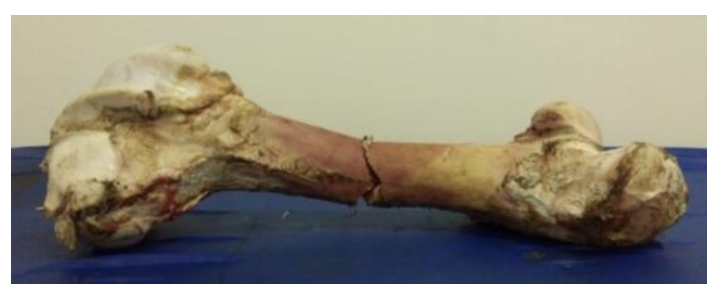

(a)

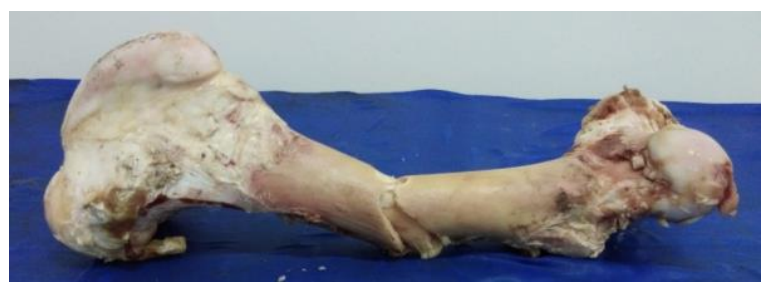

(c)

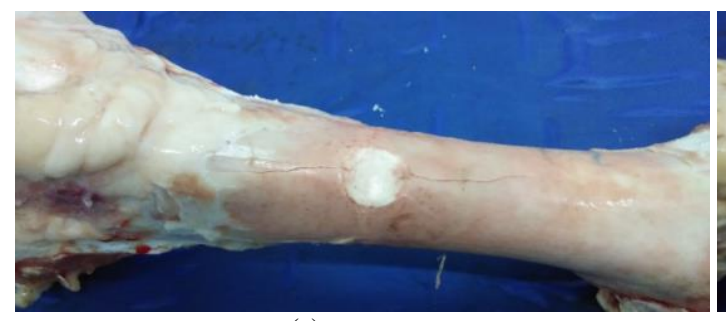

(e)

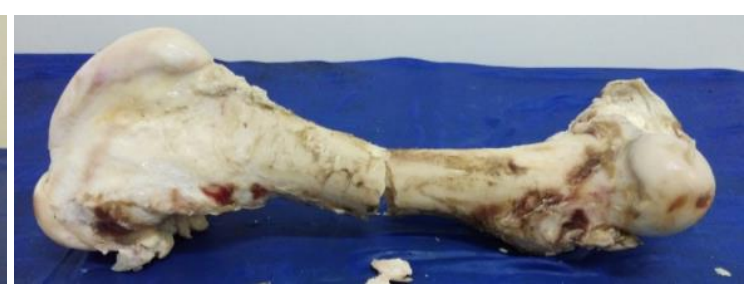

(b)

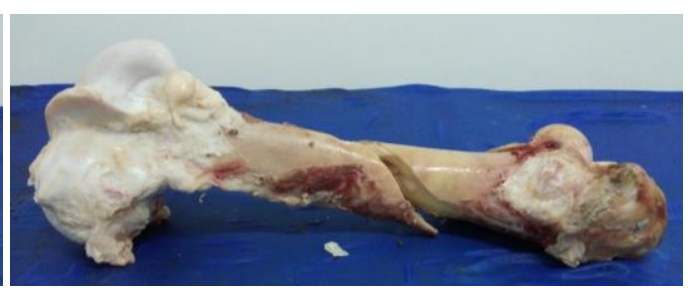

(d)

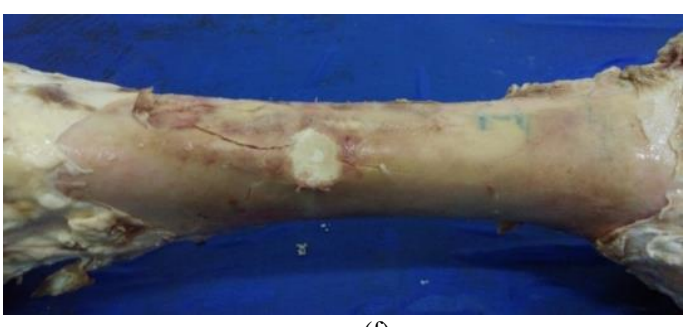

(f)

Fig. 8: Failure characteristics of femur under different loading rates; (a) $90 \mathrm{~mm} / \mathrm{min}$; (b) $10 \mathrm{~mm} / \mathrm{min}$; (c) $5 \mathrm{~mm} / \mathrm{min}$; (d) $1 \mathrm{~mm} / \mathrm{min}$; (e) $0.5 \mathrm{~mm} / \mathrm{min}$; (f) $0.1 \mathrm{~mm} / \mathrm{min}$ 


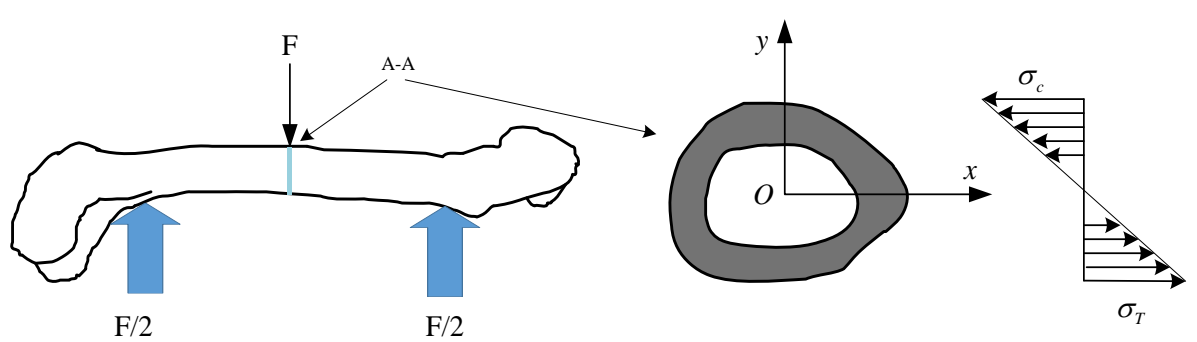

(a)
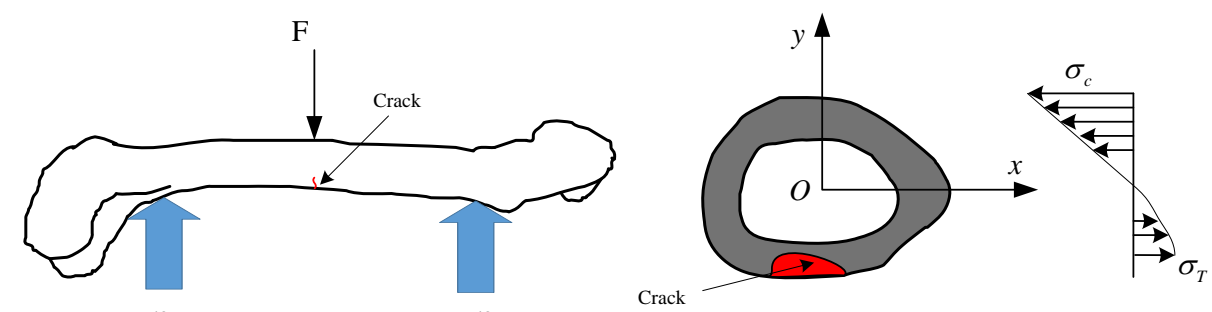

(b)
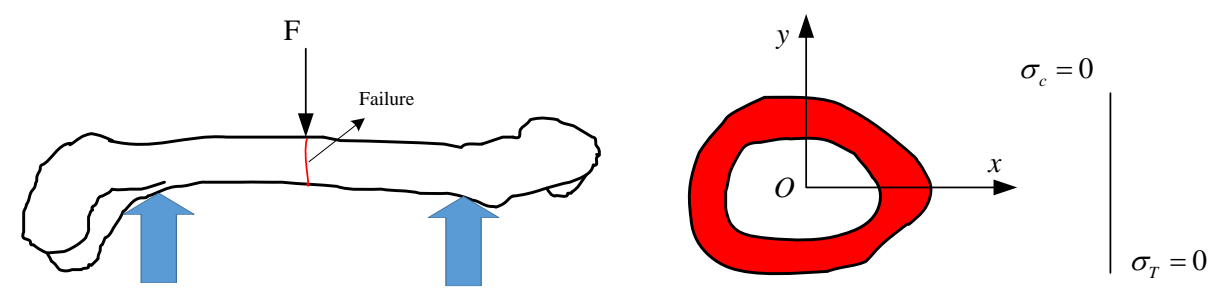

$F / 2$

F/2

(c)

Fig. 9: Evolution process of tensile failure mode; (a) Instant contact force; (b) Cracks appear; (c) Cracks coalescence to overall failure

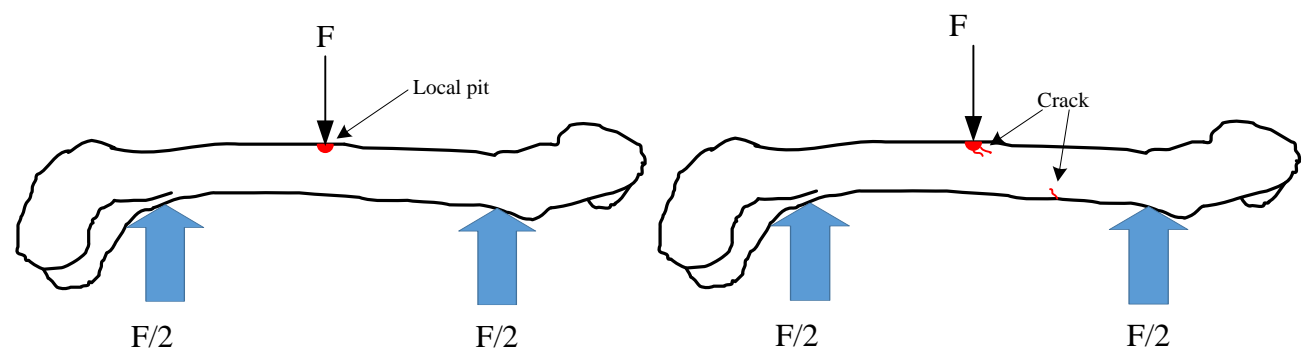

(a)

(b)

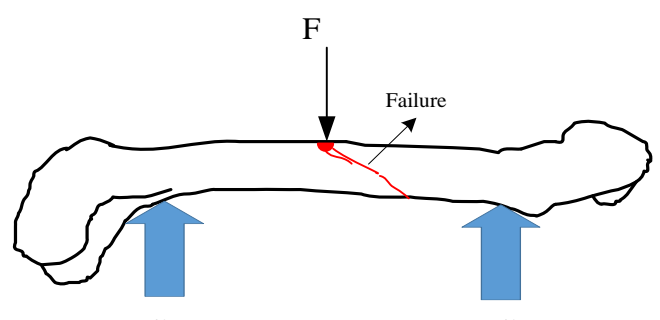

F/2

(c)

Fig. 10: Evolution process of shear failure model; (a) Local press-in; (b) Cracks appear; (c) Shear crack coalescence to the overall failure 
As for shear failure mode, the bending deformation of the whole bovine bone can be divided into three stages Fig. 10: Press-in stage, press-in bending stage and collapse stage. In the press-in stage, the bone deformation of the contact area between the loading roller and bone is mainly partial press-in and the overall bending deformation is small. In the press-in bending stage, with the increase of the degree of local pitting of the loading roller, the section bending stiffness $(E \cdot I)$ decreases, the overall bending deformation of the bone structure increases and the partial press-in and the overall bending deformation develop together. When the local bearing capacity of the bone structure reaches the maximum value, the local press-in amount does not increase; the deformation of bone structure turns to the overall bending and the bending stiffness of the section decreases with the increase of the turning angle $(\phi=$ $M /(E \cdot I))$. The shear failure mode has both press-in and bending problems, which make the theoretical maximum stress not to appear in the middle of the span.

For the wedge-shaped local failure mode, due to the low loading rate, the microstructure of the bone have sufficient time to compress and slide along the lamellar layers, forming a local pitting with micro-cracks. The local pitting of the bone changes the force state, so that the load is applied to the middle of the upper edge of the bone through the semicircle. The applied forces actually on the bone show a horizontal force of amplitude $P / \pi$ and a vertical $P$ shown in Fig. 11. The two horizontal forces are in equilibrium with each other to generate tensile stress in the small area where they act (Su et al., 1997). These microcracks begin to grow and expand longitudinally along the upper edge of the bone until they fractures as the force increases Fig. 12. Due to the low loading rate, the depth of the local pitting is large. This mode mainly consists of two stages: The press-in stage and the local tensile cracking stage of the upper edge of the hollow bone.

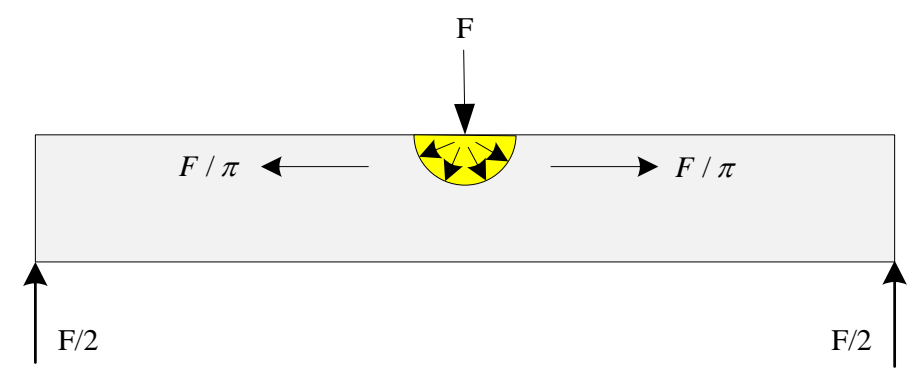

Fig. 11: Load distribution by wedge influence

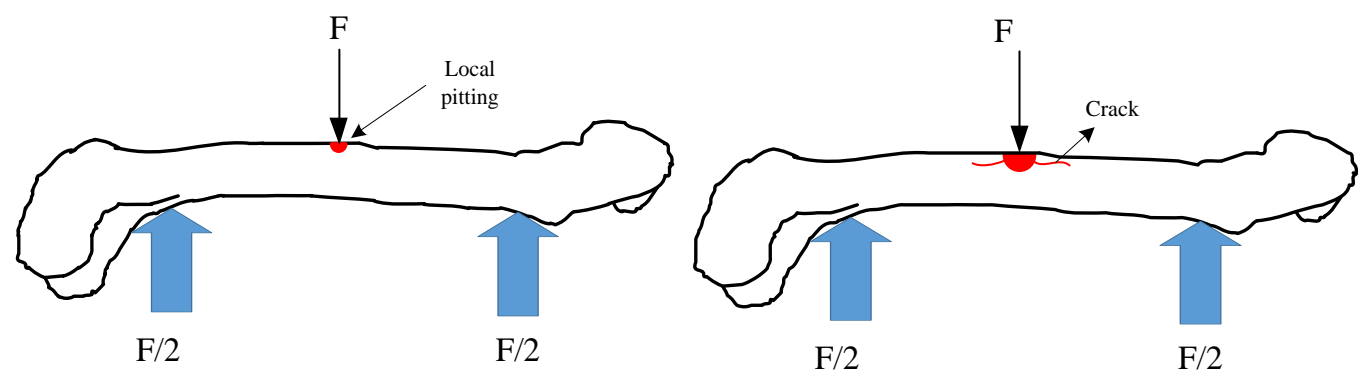

(a)

(b)

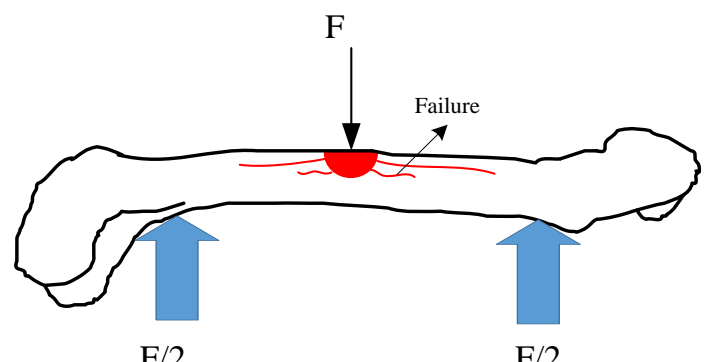

(c)

Fig. 12: Evolution process of wedging failure mode; (a) Local indentation; (b) The appearance of cracks; (c) Crack propagation to overall failure 
From the perspective of microstructure, bone is a composite material composed of mineral crystals and collagen fibers. The mineral crystals of bone are distributed between different collagen fibers and formed by mineralization outside the fibers. Therefore, bone structure can be regarded as a fiber-reinforced composite material similar to reinforced concrete. Compared with collagen fibers, mineral crystals play a dominant role for the crack development. Bending failure modes and their crack section profiles are different under different loading rates as shown in Fig. 13. Crack often begins with internal or surface micro-defects (such as microcracks and voids). The tensile cracks often appear in the weak layer of the bone. The damage caused by compression mainly originates from the intra-pore pores. When the damage reaches a certain extent, local microcracks are initiated. The faster the loading rate, the shorter the crack growth time will be. The tensile strength of bone is only about $50 \%$ of the compressive strength and the bone is destroyed immediately once the tensile crack occurs at a high loading rate. When the loading rate is slow, the micro-cracks in the bone has sufficient time to slide, resulting in enough micro-cracks until the failure, showing a large deformation and the bone beam is in the ductile zone.

\section{Relationship Between Flexural Strength of the Whole Bone and Loading Rate}

It is great significance for understanding the damage mechanism of the whole bone and the prediction and protection of damage by loading rate in the biomechanical field. The mechanical properties of bone have a certain relationship with the loading rate. The force-displacement curves of each sample from the three point bending tests are shown in Fig. 14.

From loading to failure, the bending deformation of the whole bovine bone includes three stages of elasticity, crack propagation and failure at different loading rates. At the initial stage of loading, the stress on the cross section of the bone is small and the influence of microcracks in the bone is also small and the bending deformation is basically elastic. The cross section of the beam remains flat after bending and the stress and strain remain linear. The stress distribution of compression zone and the tension zone is triangular (Fig. 9a).

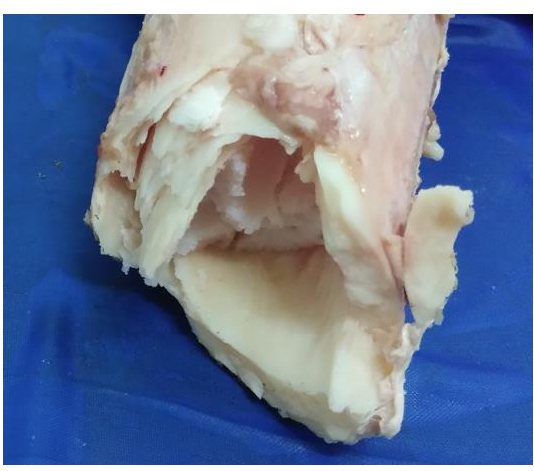

(a)

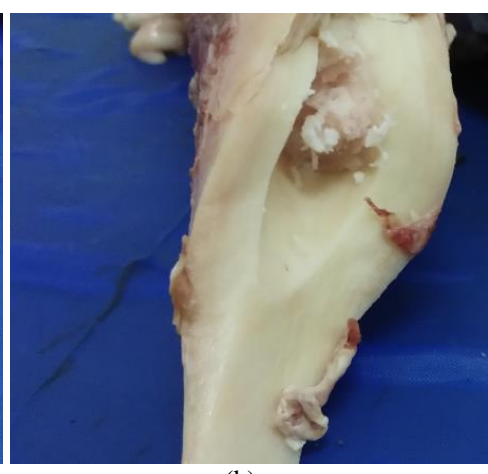

(b)

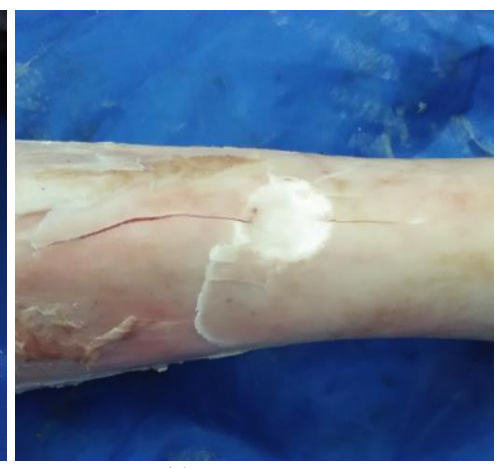

(c)

Fig. 13: Different types of crack section in three-point bending test; (a) Breaking failure; (b) Shear failure; (c)Wedge local failure

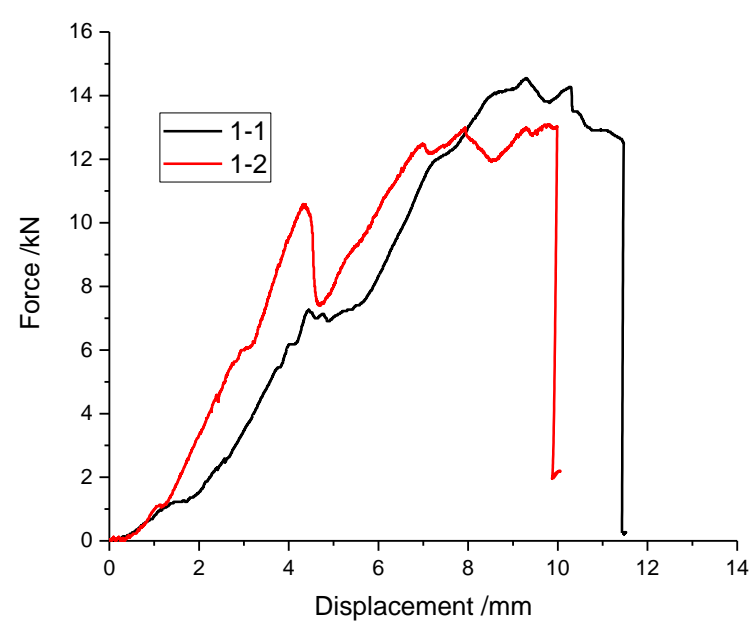

(a)

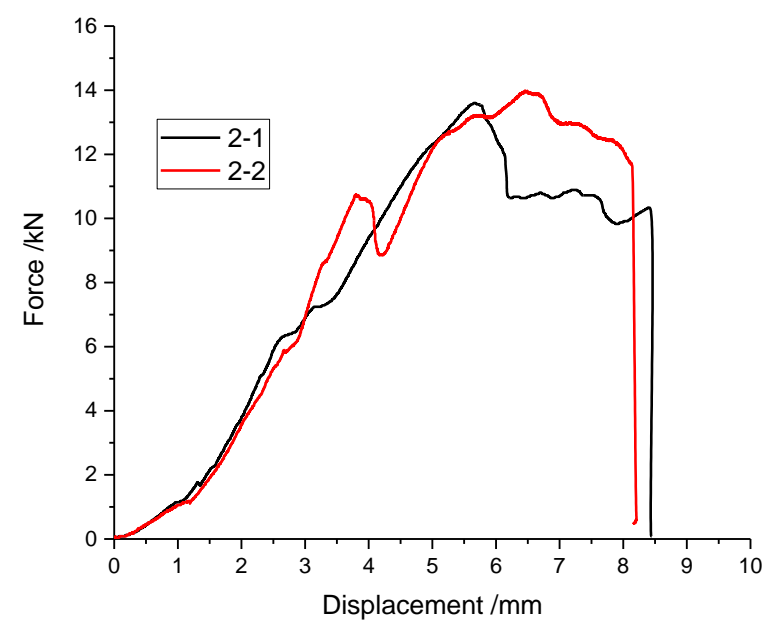

(b) 


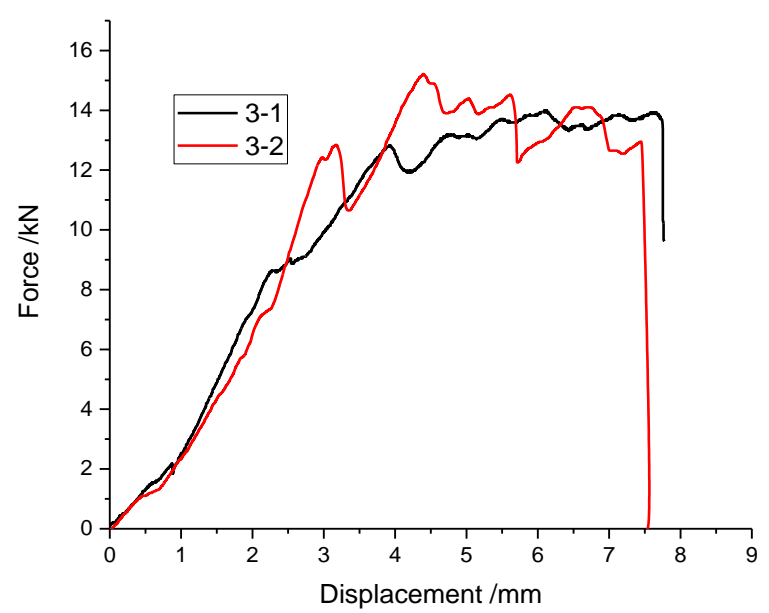

(c)

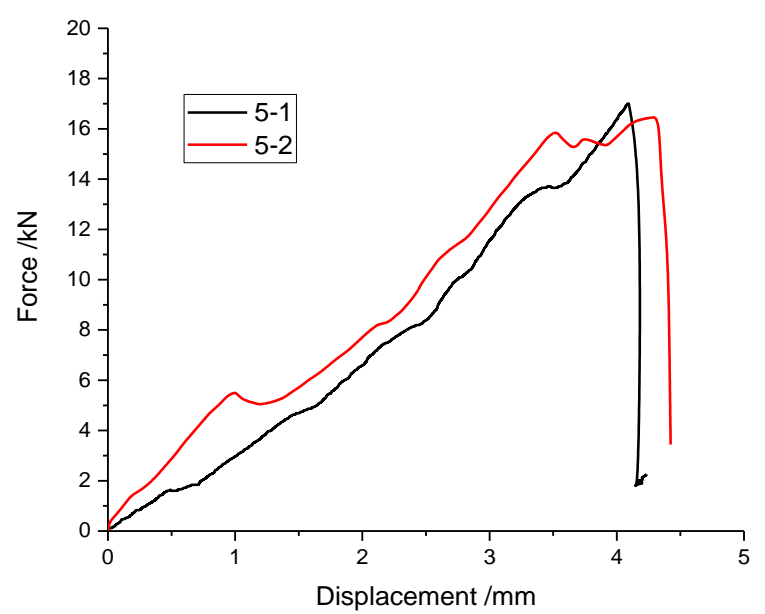

(e)

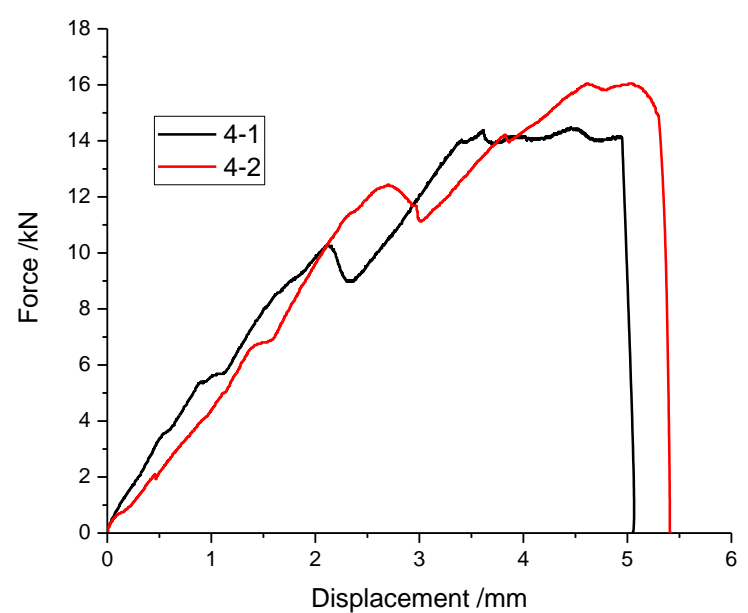

(d)

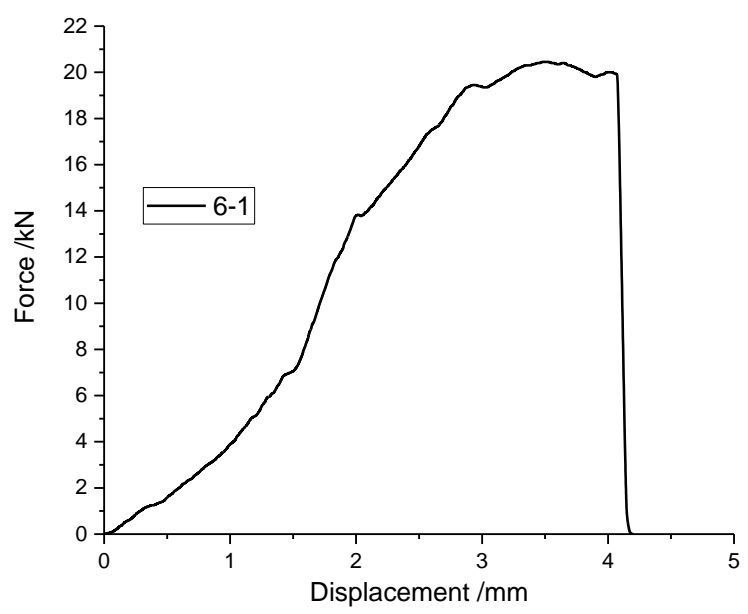

(f)

Fig. 14: Force-displacement curve at different loading rates; (a) $0.1 \mathrm{~mm} / \mathrm{min}$; (b) $0.5 \mathrm{~mm} / \mathrm{min}$; (c) $1 \mathrm{~mm} / \mathrm{min}$; (d) $5 \mathrm{~mm} / \mathrm{min}$; (e) 10 $\mathrm{mm} / \mathrm{min}$; (f) $90 \mathrm{~mm} / \mathrm{min}$

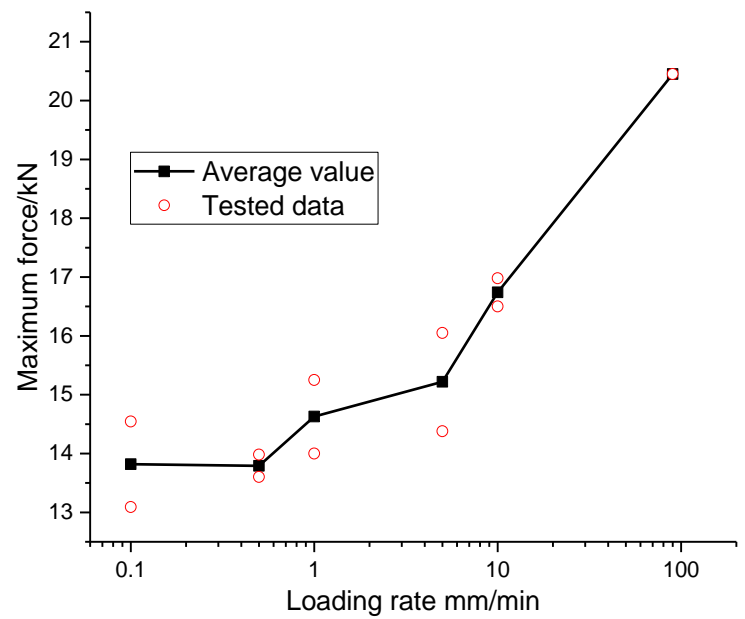

Fig. 15: Relationship between the bending strength and loading rate

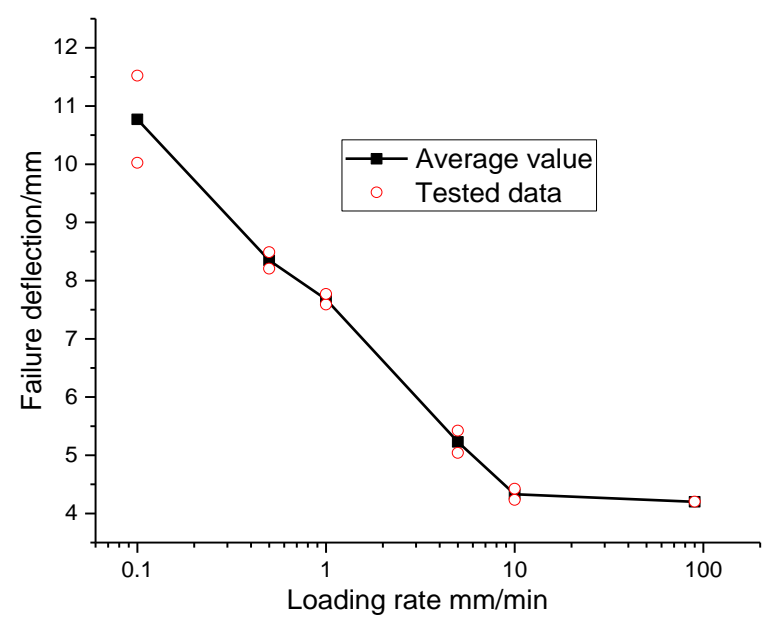

Fig. 16: Relationship between the failure deflection and loading rate 
In the crack propagation stage, the micro-cracks in the bone gradually expand and extend and new cracks appear. Since the tensile strength is smaller than the compressive strength of the bone, the micro-cracks in the tension zone expand first. When the crack spreads to a certain extent, local failure occurs in the bone and the internal stress of the bone is adjusted in the form of force-displacement curve fluctuation. After that, the crack continues to expand and the curve appears to fluctuate many times. When the loading rate is higher than $10 \mathrm{~mm} / \mathrm{min}$, the crack propagation lasts for a short time and brittle failure will occur soon when the elastic deformation reaches the peak strength. In the third stage, many small cracks develop to form the macroscopic crack and the bone strength decreases rapidly with the increase of load and then the bone is broken.

For the whole bone bending test at different loading rates, the force-displacement characteristics of femoral bone are basically similar. When the maximum force is reached, the force begins to decrease with the increase of displacement, which is due to the damage of bone material. Taking the point corresponding to the maximum force in the force-displacement curve as the failure point, the changes of bending strength and failure deflection with different loading rate are shown in Fig. 15 and 16. It can be seen that the bending strength increases gradually with the increase of loading rate, while the failure deflection tends to decrease gradually with loading rate. When the loading rate is $0.1 \mathrm{~mm} / \mathrm{min}$, the failure force is 13.82 $\mathrm{kN}$, while when the loading rate is $90 \mathrm{~mm} / \mathrm{min}$, the failure force is $20.45 \mathrm{kN}$ and the strength is increased by about $48 \%$. Comparing with the failure force at loading rate of 0.1 and $0.5 \mathrm{~mm} / \mathrm{min}$, there is no obvious change between them. The main reason is that the slower loading rate, the more stable the bending strength of the bone. After that, the bending strength increases more obviously with the increase of loading rate. When the loading rate is $0.1 \mathrm{~mm} / \mathrm{min}$, the failure deflection is $10.77 \mathrm{~mm}$ and the time-dependent failure appears in the bone with an overall performance of ductile failure. The sound of the rupture is small and lasts longer. When the loading rate is more than 10 $\mathrm{mm} / \mathrm{min}$, the failure deflection is $4.33 \mathrm{~mm}$ with an overall performance of brittle failure and the fracture sound of bone is large so that earplugs need to be worn during the test.

\section{Brittleness Evaluation of Bone}

Brittleness is an important index of bone quality, which can be evaluated by the bending test. In this study, the deformation before the peak point of forcedisplacement curve is assumed to be elastic and some parameters can be calculated by bending failure theory. According to Equation (6)-(8), the fracture stress, fracture strain and flexural modulus of bone can be calculated to reflect the brittleness characteristics of bone and understand the mechanisms of bone damage and sport fracture. For the whole bone bending test, the whole bone is not ideally straight beam and its cross-section are unequal. In this study, the whole bone is simplified as a straight beam with equal thickness of circular or elliptical annular cross section to reflect the bending mechanical properties for the whole bone Fig. 1. Figure 17 shows the relationship between bone fracture stress and loading rate. The results show that although there is a certain fluctuation between fracture stress and loading rate, the overall trend is that fracture stress increases with the increase of loading rate. In addition, the fracture stress predicted by elliptic section method is much larger than that predicted by circular section method.

Figure 18 shows the relationship between bone strain and loading rate. It can be seen that the bone strain gradually decreases with the increase of the loading rate and the decreasing rate tends to decrease gradually. The strain range of the whole bone obtained by elliptic section method is $2-6 \%$, while that obtained by circular section method is $3.5-10 \%$. According to the brittleness concept of engineering materials (Shu et al., 2018), the brittleness refers to the property that materials fail with small deformation after force. The relative deformation amount or failure strain less than $3 \%$ is called brittle failure and the failure strain greater than $5 \%$ is called time-dependent failure. In the strain range of $3-5 \%$, the failure property is in the transition state. According to the above tested results, the failure property of bone is as a visco-elastic-brittle material. The whole bone shows a brittle failure when the loading rate is greater than $5 \mathrm{~mm} / \mathrm{min}$. It proves that the strain predicted by elliptic section method is more in line with the definition of brittleness of engineering materials (Shu et al., 2018).

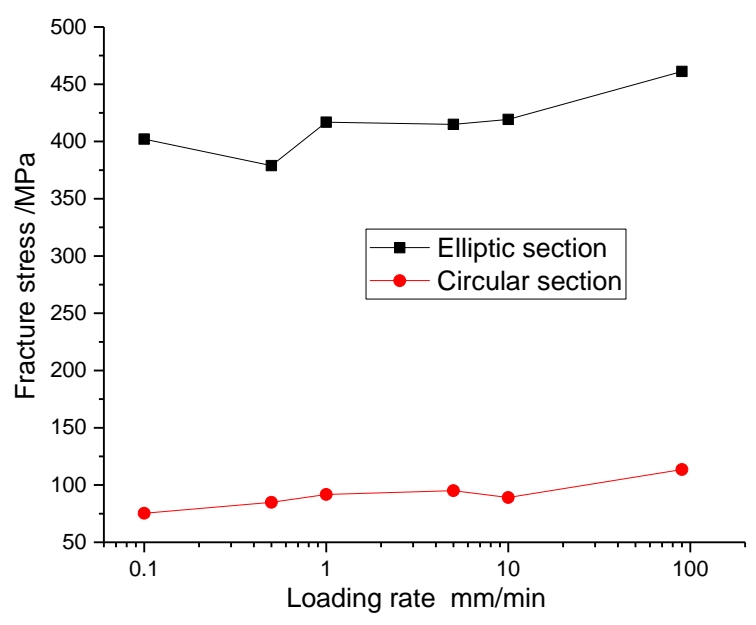

Fig. 17: Relationship between fracture stress and loading rate 


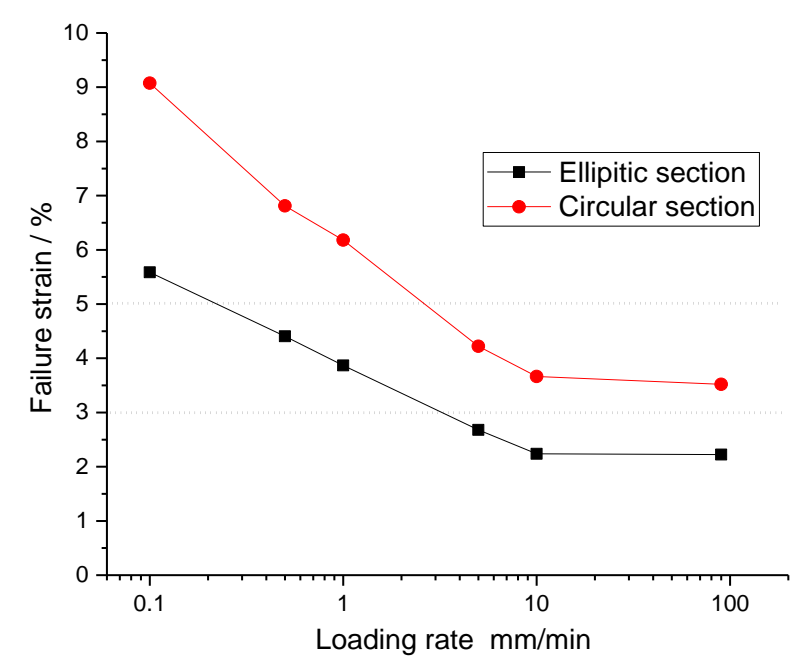

Fig. 18: Relationship between failure strain and loading rate

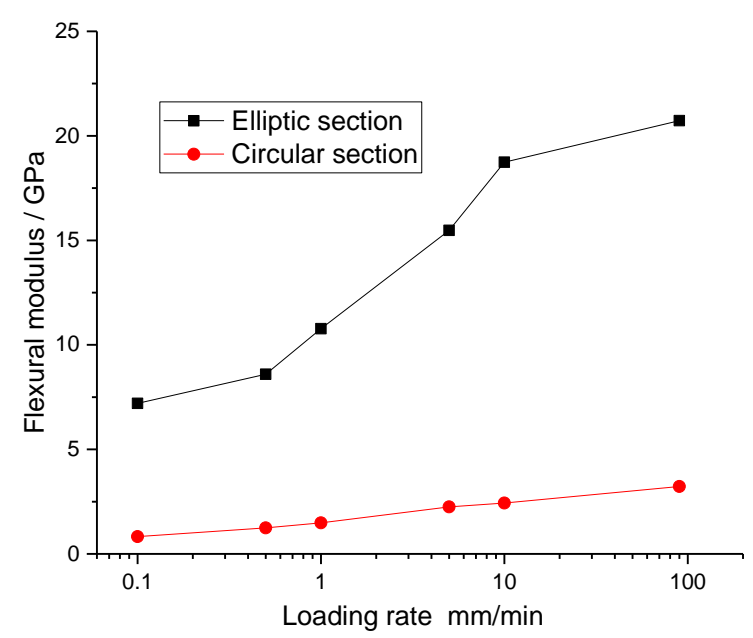

Fig. 19: Relationship between flexural modulus and loading rate

Figure 19 shows the relationship between flexural modulus of the bone and loading rate. It can be seen that the flexural modulus of the bone gradually increases with the increase of the loading rate and its regularity is good. According to the theory of solid mechanics, under a certain stress level, the smaller the deformation is when the material fails, the higher the brittleness of the material is, that is, the greater the brittleness of the material with larger elastic modulus. When the Poisson ratio is small, the material is less prone to lateral direction and more prone to failure.

Through the above analysis, the mechanical properties of the whole bone are significantly affected by the loading rate and cannot be ignored. In addition, the mechanical properties of human bones in different regions, races, ethnic groups and even different genders and ages are quite different, especially the changes in nutritional conditions and food structure (McCalden et al., 1993; Zhu et al., 2006). The mechanical properties of bones also change. At present, there is no unified biomechanical evaluation standard and it is necessary to continue experimental research in the future.

\section{Conclusions}

For bending test of femoral bone, the loading rate has a significant influence on the failure model and mechanical parameters. Based on a series of three point bending tests of the whole bovine bone, the following conclusions can be drawn:

(1) The loading rate affects the bending failure process of femoral bone and the failure mode can be divided into three types: Breaking failure, shear failure and wedgeshaped local failure. When the loading rate is high, the bone sample will immediately collapse after reaching the ultimate force. When the loading rate is low, the bone will slowly rupture after the sample reaches the ultimate force

(2) The deformation of bone under different loading rates basically experienced three stages: Elasticity, crack propagation and failure. When the loading rate is low, the non-linear deformation stage is more obvious and lasts longer. When the loading rate is higher than $10 \mathrm{~mm} / \mathrm{min}$, the crack propagation stage lasts for a short time and then the brittle failure of the sample will occur soon

(3) The loading rate has a significant effect on the mechanical properties of the whole bone. The greater the loading rate is, the smaller the failure strain is, the greater the flexural modulus of the whole bone is and the more brittleness of the bone is

(4) Due to the irregularity of geometry and the complexity of bone material (Khor et al., 2018), the proposed model has some limitations and suitable theoretical model of whole bone still need to be further studied. The degree of research on bone mechanics and damage characteristics is relatively low and there are many factors affecting the biomechanical properties of bones. The establishment of quantitative relationship between external load and bone damage and finite element analysis need to be further studied

\section{Acknowledgment}

The authors gratefully acknowledge the support from the Teaching Research Foundation of Hubei Province, China (Grant No. 2018285), China Innovation and Entrepreneurship Training Fund for College Students (Grant No. 2018157) and Basic scientific research business expenses of provincial undergraduate universities in Heilongjiang Province (Grant No. 2020YTW-T-03). 


\section{Author's Contributions}

Hua Li: Conceptualization, Methodology, WritingOriginal draft preparation.

Rujie Gao: Data curation, Conducting experiments.

Ruiqi Li: Data curation, Conducting experiments.

Xia An: Conducting experiments.

Yufeng Zhao: Conducting experiments.

All authors read and approved the final manuscript.

\section{Ethics}

The authors declare their responsibility for any ethical issues that may arise after the publication of this manuscript.

\section{Conflict of Interest}

The authors declare that they have no competing interests. The corresponding author affirms that all of the authors have read and approved the manuscript.

\section{References}

Ayagara, A. R., Langlet, A. and Hambli, R. (2019). On dynamic behavior of bone: Experimental and numerical study of porcine ribs subjected to impact loads in dynamic three-point bending tests. Journal of the mechanical behavior of biomedical materials, 98, 336-347.

Bai, S., Li Z.Q. and Jing, H. (1999). Theoretical analysis of bone damage mechanism in sports. J. Xi'an Inst. Phys. Educ, 4, 93-95.

Chen, Y., Bai B. and Gu, D.Y. (2006). Effects of loading rate on three-point bending test of rat femoral. Acad. J. Guangzhou Med. Coll, 34, 56-58.

Chen, Y., Yang, D., Ma, Y., Tan, X., Shi, Z., Li, T. and $\mathrm{Si}$, H. (2015). Experimental investigation on the mechanical behavior of bovine bone using digital image correlation technique. Applied bionics and biomechanics, 2015.

Cui, W. and Liu, C.L. (1997). Basic bone biomechanics (I). Chinese J. Osteoporosis, 3, 82-85.

Cui, W. and Liu, C.L. (1998). Basic bone biomechanics (II): Selection and calculation of biomechanical indexes of animal bone. Chinese J. Osteoporosis, 4, 90-92.

Draper, E. R. and Goodship, A. E. (2003). A novel technique for four-point bending of small bone samples with semi-automatic analysis. Journal of biomechanics, 36(10), 1497-1502.

Fan, L.X. and Wang, E. (2006). Finite element analysis of man's capability at the upper end of femoral in falling (I) finite element analysis model and establishment of femoral failure criterion. Biomed. Eng. J, 23, 1028-1032.
Ferreira, F., Vaz, M. A. and Simoes, J. A. (2006). Mechanical properties of bovine cortical bone at high strain rate. Materials characterization, 57(2), 71-79.

Ferreño, D., Sainz-Aja, J. A., Carrascal, I. A., Diego, S., Ruiz, E., Casado, J. A., ... and Gutiérrez-Solana, F. (2017). Orientation of whole bone samples of small rodents matters during bending tests. Journal of the mechanical behavior of biomedical materials, 65, 200-212.

Gao, X., Chen, M., Yang, X., Harper, L., Ahmed, I. and $\mathrm{Lu}$, J. (2018). Simulating damage onset and evolution in fully bio-resorbable composite under three-point bending. Journal of the mechanical behavior of biomedical materials, 81, 72-82.

Hansen, U., Zioupos, P., Simpson, R., Currey, J. D. and Hynd, D. (2008). The effect of strain rate on the mechanical properties of human cortical bone. Journal of biomechanical engineering, 130(1).

Jungmann, R., Szabo, M. E., Schitter, G., Tang, R. Y. S., Vashishth, D., Hansma, P. K. and Thurner, P. J. (2011). Local strain and damage mapping in single trabeculae during three-point bending tests. Journal of the mechanical behavior of biomedical materials, 4(4), 523-534.

Khor, F., Cronin, D. S., Watson, B., Gierczycka, D. and Malcolm, S. (2018). Importance of asymmetry and anisotropy in predicting cortical bone response and fracture using human body model femur in three-point bending and axial rotation. Journal of the Mechanical Behavior of Biomedical Materials, 87, 213-229.

Kourtis, L. C., Carter, D. R. and Beaupre, G. S. (2014). Improving the estimate of the effective elastic modulus derived from three-point bending tests of long bones. Annals of biomedical engineering, 42(8), 1773-1780.

van Lenthe, G. H., Voide, R., Boyd, S. K. and Müller, R. (2008). Tissue modulus calculated from beam theory is biased by bone size and geometry: Implications for the use of three-point bending tests to determine bone tissue modulus. Bone, 43(4), 717-723.

Liebschner, M. A. (2004). Biomechanical considerations of animal models used in tissue engineering of bone. Biomaterials, 25(9), 1697-1714.

McCalden, R. W., McGeough, J. A. and Barker, M. B. Court-Brown CM. (1993). Age-related changes in the tensile properties of cortical bone. The relative importance of changes in porosity, mineralization, and microstructure. J Bone Joint Surg Am, 75(8), 1193-1205.

Mumtaz, H., Dallas, M., Begonia, M., Lara-Castillo, N., Scott, J. M., Johnson, M. L. and Ganesh, T. (2020). Age-related and sex-specific effects on architectural properties and biomechanical response of the C57BL/6N mouse femur, tibia and ulna. Bone Reports, 100266. 
Pithioux, M., Subit, D. and Chabrand, P. (2004). Comparison of compact bone failure under two different loading rates: Experimental and modelling approaches. Medical engineering and physics, 26(8), 647-653.

Raftopoulos, D., Katsamanis, E., Saul, F., Liu, W. and Saddemi, S. (1993). An intermediate loading rate technique for the determination of mechanical properties of human femoral cortical bone. Journal of biomedical engineering, 15(1), 60-66.

Ridha, H. and Thurner, P. J. (2013). Finite element prediction with experimental validation of damage distribution in single trabeculae during three-point bending tests. Journal of the Mechanical Behavior of Biomedical Materials, 27, 94-106.

Shu, K.T. Jia S.P. and Gao, Y. (2018). Improved method for evaluation of brittleness of cap rock based on full stress-strain curve. J. Guangxi Univ, 43, 248-260.

Su, X.P., Lin G. and Song, X.H. (1997). The valuation of the bending strength testing method on brittle materials. J. Wuhan Univ. Technol, 19, 34-37.
Sun, L. W., Li, S., Yang, X., Pu, F., Qi, H. and Fan, Y. (2018). Contribution of bone micromechanical behavior beyond lamellar length scale to the macroscopic bone quality of hind limb unloading rats. Acta Astronautica, 152, 468-473.

Szabó, M. E., Taylor, M. and Thurner, P. J. (2011). Mechanical properties of single bovine trabeculae are unaffected by strain rate. Journal of biomechanics, 44(5), 962-967.

Szabó, M. E. and Thurner, P. J. (2013). Anisotropy of bovine cortical bone tissue damage properties. Journal of biomechanics, 46(1), 2-6.

Yang, J. (2016). The effect of formalin fixation on the biomechanical properties of bovine femoral cortical bone. MSc Thesis, Hunan University, china.

Zhu, L., Zhao H.P. and Song, Y.L. (2006). Experimental study on mechanical properties of femoral bone in takin. J. Tsinghua Univ, 46, 301-304. 